\title{
Atypical presentation of CVST as bradycardia and psychosis
}

\author{
Neeta Sagar Bobba ${ }^{1^{*}}$, Sumati Kumatgi², Chytanya Deepak Ponangi ${ }^{3}$ \\ ${ }^{1}$ MBBS, ${ }^{2} \mathrm{DPM},{ }^{3}$ Senior Consultant, ${ }^{\mathbf{1}}$ Sri Ramachandra Medical College and Research Institute, Chennai, Tamil Nadu, ${ }^{2,3}$ ASHA Hospital, \\ Hyderabad, Telangana, India \\ *Corresponding Author: Neeta Sagar Bobba \\ Email: neetasagar11@gmail.com
}

\begin{abstract}
Cerebral venous sinus thrombosis (CVST) causing cerebral infarction is noted in the younger population in India and other countries, with a multitude of varying features at presentation. Typical features from mass effect due to acute and subacute complications of CVST, characteristically present with signs of neurological deficits due to raised intracranial pressure. In rare instances, patients afflicted with CVST present with isolated neuropsychiatric symptoms. We delve into this case of a middle aged woman with subacute complications whose neurological features were masked, with the sole findings of behavioral disturbance and bradycardia, who had no known previous documented psychiatric illness.
\end{abstract}

Keywords: Atypical, Cerebral, Venous, Thrombosis, Bradycardia.

\section{Introduction}

Cerebral venous sinus thrombosis (CVST) is an atypical cause of cerebral infarction, as strokes tend to stem frequently from arterial diseases. CVST tends to be more challenging to diagnose due it's widely variable clinical presentation. The incidence of CVST is thought to be higher in India than other countries, but estimated incidence is still relatively unknown. ${ }^{1,2}$ Predisposing factors for CVST include hypercoaguable states, which may be either hereditary or acquired, such as genetic thrombophilias, systemic inflammatory diseases, puerperium, or infection in the CNS. ${ }^{3}$ The mean age of afflicted patients was found to be 31.3 years, ${ }^{4}$ with typical presentation of features of raised intracranial pressure (ICP), including headache, seizures, motor weakness and visual disturbances. ${ }^{5}$ Documentation of behavioral changes at presentation have also been historically reported, with features of delirium, anxiety, and depression, although rarer when compared to neurologic deficits. ${ }^{6}$ This middle aged patient's lack of classic features, presenting only with behavioral disturbances (in the absence of known psychiatric illness) and bradycardia with mass effect from edema and subacute hemorrhagic infarct makes this case unique.

\section{Case Report}

We report a case of a 55 year old woman hailing from a rural setting in South India. She was brought by her husband and son to the psychiatry outpatient department, with complaints of decreased sleep, fearfulness, suspiciousness, disorganized speech, agitation, increased aggression, along with the inability to recognize her relatives for the past 3 days. Patient had no known past history of trauma, loss of consciousness, headache, visual disturbances, depression, mania, co-morbid psychiatric illness, or suicidality.

Patient has a significant past history of consuming alcohol, spanning over the last 20 years. Patient initially consumed toddy fortnightly, which gradually progressed to a weekly basis in the initial decade. Over the past ten years, patient regularly consumed $200-400 \mathrm{ml}$ of toddy on a daily basis, with brief interspersed periods of reluctant abstinence for 4 to 5 days. Patient experienced no symptoms of withdrawal, seizures, pain abdomen, jaundice or hematemesis during aforementioned timeframes. Patient reportedly decreased alcohol consumption abruptly over the past 2 weeks, her last alleged drink was 3 days ago, after which she started to experience present symptoms for which she was brought to the hospital. Patient has been regularly chewing tobacco with betel nut for the past 30 years, no known history of intravenous drug abuse. Patient had no past history of hypertension, diabetes, epilepsy, thyroid, autoimmune disease, hematologic-oncologic disease, previous surgeries or invasive procedures. Patient's brother and husband both have a significant history with alcohol dependence syndrome, her brother also has a history of epilepsy.

Vitals were as follows; pulse: 40 beats/min, BP: 100/70 $\mathrm{mm} \mathrm{Hg}$, respiratory rate: 17 breaths/min, temperature: 97.8 ${ }^{\circ} \mathrm{F}$ SpO2: $87 \%$. On general examination, patient appeared to be of thin build, unkempt, mildly tremulous and restless while constantly gesturing in the air. Increased psychomotor activity was observed. Mental status examination: Patient had altered consciousness, was disoriented to time and place, and oriented to person only, with decreased attention and concentration and recent memory impairment. Speech was spontaneous, irrelevant and incoherent. Thought form was impaired, with presence of paranoia, with an insight of grade $1 / 6$.

Systemic examination: CNS: 1. Cranial Nerves: Pupils were equal, round reactive to light, with intact ocular movement, no observed deviation or nystagmus. Face was symmetric at rest with intact sensation throughout. No dysarthria, full strength in sternocleidomastoid and trapezius bilaterally. 2. Motor: Muscle bulk and tone was normal with a strength of 5/5 in all four extremities, both proximally and distally, no pronator drift observed. $2+$, symmetric reflex 
observed bilaterally in the biceps, triceps, brachioradialis, patella and achilles. Plantar response was flexor bilaterally. 3. Sensory: Sensation was intact to touch, pinprick, vibration and proprioception throughout. No evidence of Romberg sign was observed. 4. Coordination: Normal finger to nose and heel to shin, no observed truncal ataxia, tremor or dysmetria observed. 5. Gait: Narrow based with normal stride length with arm swing bilaterally, She was able to walk on heels and toes in tandem. Cardiovascular System: S1, S2 audible with no extra heart sounds, no carotid bruit. Respiratory System: Normal vesicular breath sounds heard. Abdomen: Soft, Bowel sounds present.

A differential diagnosis of Alcohol Dependence syndrome with withdrawal delirium tremens and organic psychosis was made in light of the above findings. Patient was started on Inj.Lorazepam $2 \mathrm{mg}$ IM immediately. Serum electrolytes, complete blood count and thyroid profile were within reference lab range, random blood glucose: 129 $\mathrm{mg} / \mathrm{dl}$. Patient was then initiated on Inj. Thiamine $150 \mathrm{mg}$
OD IM, Tab. Thiamine 100mg BD, Tab. Pantoprazole 40mg OD, T. Risperidone $1 \mathrm{mg}$ OD. After symptomatic improvement, patient underwent ECG, and 2D ECHO imaging which revealed no abnormality, patient was then scheduled for an MRI without contrast.

MRI revealed hemorrhagic venous infarct in the subacute stage in the left posterior parieto-temporal lobe with mass effect in the form of effacement of ipsilateral ventricle, basal cisterns and midline shift of $4 \mathrm{~mm}$ to the right. There was diffuse cerebral edema involving the left supratentorial cerebral parenchyma. MR venography revealed complete absence of blood flow in left transverse and sigmoid sinuses with patchy flow in straight sinus along with the presence of dural venous thrombosis. Post MRI review, the diagnosis of CVST was made and patient was immediately shifted to an ICU at a general hospital, to seek appropriate treatment to prevent recurrence of hemorrhage and potential embolism.

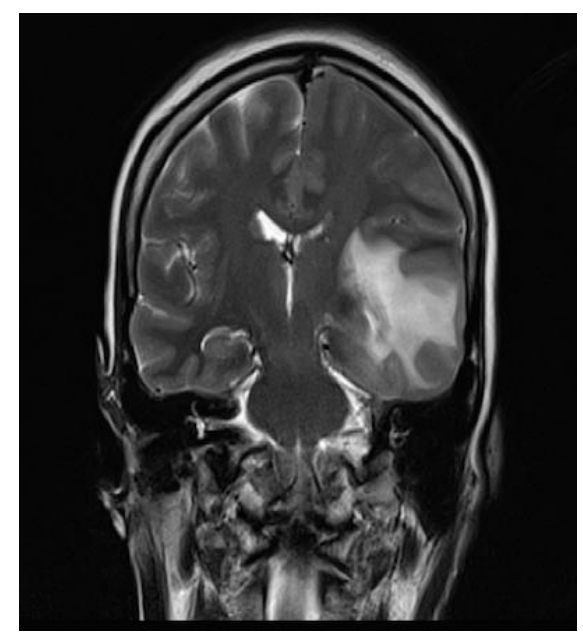

Fig. 1: MRI demonstrating mass effect noted in form of mild effacement of ipsilateral ventricle and basal cisterns with midline shift of $4 \mathrm{~mm}$ to the right. There is mild left uncal herniation
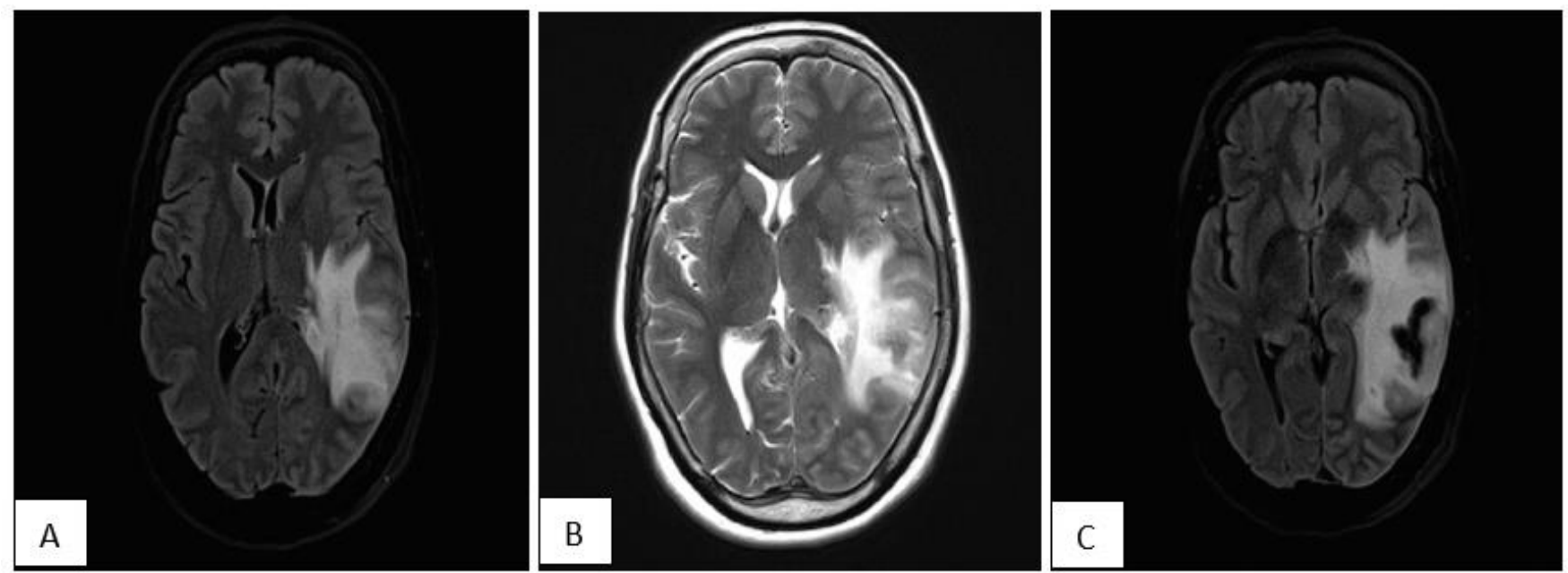

Fig. 2: Altered signal intensity with marked adjacent edema is noted in the left posterior parieto-temporal lobe. It is hypodense on T2W images, hyperintense on T1W images and shows blooming on GRE images. Focal areas of restriction noted on DW image 


\section{Discussion}

The anatomical structure of venous outflow in the brain is encompassed by sinuses (spaces between meningeal dural membranes and periosteum) and veins, only the latter are regarded as true blood vessels, which are further subdivided into superficial and deep veins. The major sinuses coalesced with cortical veins to form an integrated venous drainage system include the superior (responsible for CSF absorption via arachnoid granules) and inferior sagittal sinus which drain the superficial surfaces of both cerebral hemispheres. The straight sinus, cavernous sinus, and lateral (transverse and sigmoid) sinus, which ultimately drain into the respective left and right internal jugular veins; forming the deep system.

Consequences of venous obstruction include decreased cerebrospinal fluid absorption, decreased capillary perfusion, venous and capillary rupture due to increased pressure, or blood brain barrier disruption, resulting in vasogenic, cytotoxic edema and ischemia which may initially be compensated by cerebral vein dilation via collaterals temporarily. When the aforementioned effects are not addressed within timeframe, features of raised intracranial pressure, characteristically present as headache, focal deficits (weakness of limbs, aphasia) seizures, altered consciousness, psychosis or coma. ${ }^{7}$

Complications include venous hemorrhagic infarct, transtentorial herniation ${ }^{8}$ and sepsis. The patient's presentation of bradycardia (change in pulse, respiratory rate, and blood pressure are late signs of raised ICP usually associated to ischemia), in the absence of other typical signs of raised ICP and psychosis in this case, indicates the importance of establishing thorough assessment and examination early on.

\section{Conclusion}

CVST with progression to subacute venous hemorrhagic infarct is associated with a poor prognosis. Accurate neuroimaging, such as MR venography along with methodical therapeutic management with anticoagulation ${ }^{9}$ with early diagnosis reduces the mortality and morbidity. ${ }^{10}$ CVST has a favorable outcome if diagnosed and treated early, albeit its complexity of varying presenting signs and symptoms. This highlights the importance for clinicians to be familiar with different presentations of CVST, including isolated ICP with no focal neurological deficits, seizures or headache ${ }^{11}$ as seen in this clinical scenario.

\section{References}

1. Dash D, Prasad K, Joseph L. Cerebral venous thrombosis: An Indian perspective. Neurol India. 2015;63(3):318.

2. Ferro JM, Canho P, Stam J, Bousser MG, Barinagarrementeria F. Prognosis of cerebral vein and dural sinus thrombosis: results of the International Study on Cerebral Vein and Dural Sinus Thrombosis (ISCVT). Stroke. 2004;35(3):664-70.

3. Patil V, Choraria K, Desai N, Agrawal S. Clinical profile and outcome of cerebral venous sinus thrombosis at tertiary care center. J Neurosci Rural Pract. 2014;5(3):218.

4. Kaul S, Meena A, Mridula K, Narayan D, Ravishankar K, Suryaprabha $\mathrm{T}$ et al. Risk factors, clinical profile, and longterm outcome of 428 patients of cerebral sinus venous thrombosis: Insights from Nizam's Institute Venous Stroke Registry, Hyderabad (India). Neurol India. 2012;60(2):154

5. Dunn L. Raised intracranial pressure. J Neurol, Neurosurg Psychiatry. 2002;73(suppl 1):i23-7.

6. Ozdilek B, Zincir S, Domac F. Consideration of cerebral venous thrombosis as a cause of delirium in psychiatry clinics. Bull Clin Psychopharmacol. 2015;25(2):190-2.

7. Bousser M, Ferro J. Cerebral venous thrombosis: an update. Lancet Neurol. 2007;6(2):162-70.

8. Canhão P, Ferro J, Lindgren A, Bousser M, Stam J, Barinagarrementeria F. Causes and Predictors of Death in Cerebral Venous Thrombosis. Stroke. 2005;36(8):1720-5.

9. Raj Pulu Ishwara P, Raghavendra BS. Oral anticoagulation in cerebral venous sinus thrombosis-Experience from a tertiary care hospital in Karnataka, South India. Indian J Neurosci. 2019;5(3):113-6.

10. Saposnik G, Barinagarrementeria F, Brown R, Bushnell C, Cucchiara B, Cushman M et al. Diagnosis and management of cerebral venous thrombosis. Stroke. 2011;42(4):1158-92.

11. Coutinho J, Stam J, Canhão P, Barinagarrementeria F, Bousser M, Ferro J. Cerebral venous thrombosis in the absence of headache. Stroke. 2015;46(1):245-7.

How to cite this article: Bobba NS, Kumatgi S, Ponangi DC. Atypical presentation of CVST as bradycardia and psychosis. Telangana J Psychiatry. 2020;6(1):96-98.

\section{Acknowledgment}

Nil.

\section{Source of Funding}

Nil.

\section{Conflict of Interest}

Nil. 